\title{
A Liouville theorem for solutions of degenerate Monge-Ampère equations
}

\author{
Tianling Jin and Jingang Xiong
}

\begin{abstract}
In this paper, we give a new proof of a celebrated theorem of Jörgens which states that every classical convex solution of

$$
\operatorname{det} \nabla^{2} u(x)=1 \text { in } \mathbb{R}^{2}
$$

has to be a second order polynomial. Our arguments do not use complex analysis, and can be applied to establish such Liouville type theorems for solutions of a class of degenerate Monge-Ampère equations. We prove that every convex generalized (or Alexandrov) solution of

$$
\operatorname{det} \nabla^{2} u\left(x_{1}, x_{2}\right)=\left|x_{1}\right|^{\alpha} \quad \text { in } \mathbb{R}^{2},
$$

where $\alpha>-1$, has to be

$$
u\left(x_{1}, x_{2}\right)=\frac{a}{(\alpha+2)(\alpha+1)}\left|x_{1}\right|^{2+\alpha}+\frac{a b^{2}}{2} x_{1}^{2}+b x_{1} x_{2}+\frac{1}{2 a} x_{2}^{2}+\ell\left(x_{1}, x_{2}\right)
$$

for some constants $a>0, b$ and a linear function $\ell\left(x_{1}, x_{2}\right)$.

This work is motivated by the Weyl problem with nonnegative Gauss curvature.
\end{abstract}

\section{Introduction}

A celebrated theorem of Jörgens states that every entire classical convex solution of

$$
\operatorname{det} \nabla^{2} u(x)=1
$$

in $\mathbb{R}^{2}$ has to be a second order polynomial. This theorem was first proved by Jörgens [20] using complex analysis methods. An elementary and simpler proof, which also uses complex analysis, was later given by Nitsche [23], where Bernstein theorem for two dimensional minimal surfaces is established as a corollary. Jörgens' theorem was extended to smooth convex solutions in higher dimensions by Calabi [8] for dimension $\leq 5$ and by Pogorelov [26] for all dimensions. 
Another proof was given by Cheng and Yau [9] along the lines of affine geometry. Note that each local generalized solution of (1) in dimension two is smooth, but this is false in dimension $\geq 3$. Caffarelli [4] established the Jörgens-Calabi-Pogorelov theorem for generalized solutions (or viscosity solutions). Trudinger-Wang [27] proved that the only convex open subset $\Omega$ of $\mathbb{R}^{n}$ which admits a convex $C^{2}$ solution of (1) in $\Omega$ with $\lim _{x \rightarrow \partial \Omega} u(x)=\infty$ is $\Omega=\mathbb{R}^{n}$. Caffarelli$\mathrm{Li}$ [6] established the asymptotical behaviors of viscosity solutions of (1) outside of a bounded convex subset of $\mathbb{R}^{n}$ for $n \geq 2$ (the case $n=2$ was studied before in Ferrer-Martínez-Milán [12,13] using complex analysis), from which the Jörgens-Calabi-Pogorelov theorem follows.

In this paper, we provide a new proof of this Jörgens' theorem. Our arguments do not use complex analysis. This allows us to establish such Liouville type theorems for solutions of a class of degenerate Monge-Ampère equations. More precisely, we classify entire convex solutions of the degenerate Monge-Ampère equations

$$
\operatorname{det} \nabla^{2} u\left(x_{1}, x_{2}\right)=\left|x_{1}\right|^{\alpha} \quad \text { in } \mathbb{R}^{2},
$$

where $\alpha>-1$. The equation (2) appears, for instance, as a blowup limiting equation of

$$
\operatorname{det} \nabla^{2} u\left(x_{1}, x_{2}\right)=\left(x_{1}^{2}+x_{2}^{2}\right)^{\alpha / 2}
$$

in Daskalopoulos-Savin [10] in the study of the Weyl problem with nonnegative Gauss curvature.

In 1916, Weyl [28] posed the following problem: Given a Riemannian metric $g$ on the 2dimensional sphere $\mathbb{S}^{2}$ whose Gauss curvature is positive everywhere, does there exist a global $C^{2}$ isometric embedding $X:\left(\mathbb{S}^{2}, g\right) \rightarrow\left(\mathbb{R}^{3}, \mathrm{~d} s^{2}\right)$, where $\mathrm{d} s^{2}$ is the standard flat metric on $\mathbb{R}^{3}$ ?

Lewy [21] solved the problem in the case that $g$ is real analytic. In 1953, Nirenberg [22] gave a solution to this problem under the regularity assumption that $g$ has continuous fourth order derivatives. The result was later extended to the case that $g$ has continuous third order derivatives by Heinz [17]. An entirely different approach was taken independently by Alexandrov and Pogorelov; see [1, 24, 25].

There are also work (see [19, 14, 18, 10]) which study the problem with nonnegative Gauss curvature. Guan-Li [14] showed that for any $C^{4}$ metric on $\mathbb{S}^{2}$ with nonnegative Gauss curvature, there always exists a global $C^{1,1}$ isometric embedding into $\left(\mathbb{R}^{3}, \mathrm{~d} s^{2}\right)$; see also Hong-Zuily [18] for a different approach to this $C^{1,1}$ embedding result. Guan and $\mathrm{Li}$ asked there that whether the $C^{1,1}$ isometric embeddings can be improved to be $C^{2, \gamma}$ or even $C^{2,1}$. The problem can be reduced to regularity properties of solutions of a Monge-Ampère equation that becomes degenerate at the points where the Gauss curvature vanishes. If the Gauss curvature of $g$ only has one nondegenerate zero, the regularity of the isometric embedding amounts to studying the regularity of solutions of (3) near the origin for $\alpha=2$, and it has been proved in Daskalopoulos-Savin [10] that the solutions of (3) are $C^{2, \gamma}$ near the origin for $\alpha>0$.

A comprehensive introduction to the Weyl problem and related ones can be found in the monograph Han-Hong [16].

The main result of this paper is the following: 
Theorem 1.1. Let $u$ be a convex generalized (or Alexandrov) solution of (2) with $\alpha>-1$. Then there exist some constants $a>0, b$ and a linear function $\ell\left(x_{1}, x_{2}\right)$ such that

$$
u\left(x_{1}, x_{2}\right)=\frac{a}{(\alpha+2)(\alpha+1)}\left|x_{1}\right|^{2+\alpha}+\frac{a b^{2}}{2} x_{1}^{2}+b x_{1} x_{2}+\frac{1}{2 a} x_{2}^{2}+\ell\left(x_{1}, x_{2}\right) .
$$

Recall that every generalized solution of (1) in an open subset of $\mathbb{R}^{2}$ is strictly convex (and thus, smooth). However, this is not the case for generalized (or even classical) solutions of $\operatorname{det} \nabla^{2} u=\left|x_{1}\right|^{\alpha}$ when $\alpha>0$; see Example 4.3. And it follows from [3] that the generalized solutions of such equations with homogenous boundary condition are strictly convex.

The paper is organized as follows. To illustrate our method, in Section 2 we first present another proof of Jörgens' theorem, which only makes use of a few properties of harmonic functions. Those properties also hold in general for solutions of elliptic or even certain degenerate elliptic equations, such as a Grushin type equation shown in Section 3 that the partial Legendre transform of $u$ satisfies. In Section 4 we show that entire solutions of (2) are strictly convex and prove Theorem 1.1 .

Acknowledgements: Both authors thank Professor YanYan Li for valuable suggestions and constant encouragement. The second author was supported in part by the First Class Postdoctoral Science Foundation of China (No. 2012M520002).

\section{A new proof of Jörgens' theorem}

Proof of Theorem 1.1 when $\alpha=0$. First of all, we know that $u$ is smooth. Define $T: \mathbb{R}^{2} \rightarrow \mathbb{R}^{2}$ by

$$
T\left(x_{1}, x_{2}\right)=\left(x_{1}, \nabla_{x_{2}} u(x)\right)=:\left(p_{1}, p_{2}\right) .
$$

Clearly, $T$ is injective. Recall that the partial Legendre transform $u^{*}(p)$ is defined as

$$
u^{*}(p)=x_{2} \nabla_{x_{2}} u(x)-u(x) .
$$

Then

- $u^{*}$ is concave w.r.t. $p_{1}$ and convex w.r.t. $p_{2}$;

- $\left(u^{*}\right)^{*}=u$;

- $\Delta u^{*}=0$ in $T\left(\mathbb{R}^{2}\right)$.

Step 1: Prove the theorem under the assumption $T\left(\mathbb{R}^{2}\right)=\mathbb{R}^{2}$.

For simplicity, we will denote $\nabla_{x_{i}} u(x), \nabla_{p_{i}} u^{*}(p)$ as $u_{i}(x), u_{i}^{*}(p)$ respectively throughout the paper if there is no possibility of confusion. Since $u^{*}$ is convex w.r.t. $p_{2}$, we have

$$
u_{22}^{*} \geq 0 \quad \text { and } \quad \Delta u_{22}^{*}=0 \text { in } \mathbb{R}^{2} .
$$


It follows from Liouville theorem for entire nonnegative harmonic functions that $u_{22}^{*}=a \geq 0$ for some constant $a$. By the equation of $u^{*}$, we have $u_{11}^{*}=-a$. Hence,

$$
u^{*}=\left(-p_{1}^{2}+p_{2}^{2}\right) a / 2+b p_{1} p_{2}+\ell\left(p_{1}, p_{2}\right)
$$

for some constant $b$ and linear function $\ell$. Since $u=\left(u^{*}\right)^{*}, a>0$ and we are done.

Step 2: Prove $T\left(\mathbb{R}^{2}\right)=\mathbb{R}^{2}$.

We prove it by contradiction. Suppose that there exists $\bar{x}_{1}$ such that

$$
\lim _{x_{2} \rightarrow+\infty} u_{2}\left(\bar{x}_{1}, x_{2}\right):=\beta<+\infty .
$$

Claim: for any $x_{1} \in \mathbb{R}$,

$$
\lim _{x_{2} \rightarrow+\infty} u_{2}\left(x_{1}, x_{2}\right)=\beta .
$$

Indeed, by the convexity of $u$, for $t>0$

$$
u\left(\bar{x}_{1}, 0\right)+t \beta \geq u\left(\bar{x}_{1}, t\right) \geq u\left(x_{1}, x_{2}\right)+u_{1}(x)\left(\bar{x}_{1}-x_{1}\right)+u_{2}(x)\left(t-x_{2}\right),
$$

namely,

$$
u_{2}(x)\left(1-x_{2} / t\right) \leq \beta+\frac{1}{t}\left\{u\left(\bar{x}_{1}, 0\right)-u\left(x_{1}, x_{2}\right)-u_{1}(x)\left(\bar{x}_{1}-x_{1}\right)\right\} .
$$

Sending $t \rightarrow \infty$, we have $u_{2}\left(x_{1}, x_{2}\right) \leq \beta$. Hence, $\lim _{x_{2} \rightarrow+\infty} u_{2}\left(x_{1}, x_{2}\right) \leq \beta$. Repeating this argument with $x_{1}$ and $\bar{x}_{1}$ exchanged, we would see that $\lim _{x_{2} \rightarrow+\infty} u_{2}\left(x_{1}, x_{2}\right) \geq \beta$.

Without loss of generality, we assume that $\beta=1$. Therefore,

$$
T\left(\mathbb{R}^{2}\right)=(-\infty,+\infty) \times\left(\beta_{0}, 1\right)
$$

for some $-\infty \leq \beta_{0}<1$. Since $T$ is one-to-one and $u_{2}^{*}\left(p_{1}, p_{2}\right)=x_{2}$, we have

$$
\lim _{p_{2} \rightarrow 1^{-}} u_{2}^{*}\left(p_{1}, p_{2}\right)=+\infty
$$

i.e., for any $C>2$, there exists $\varepsilon$ (may depend on $\bar{p}_{1}$ which is arbitrarily fixed) such that $u_{2}^{*}\left(\bar{p}_{1}, p_{2}\right) \geq C$ for every $p_{2} \geq 1-\varepsilon$. By continuity of $u_{2}^{*}, u_{2}^{*}\left(p_{1}, 1-\varepsilon\right) \geq C-1$ for $p_{1} \in\left(\bar{p}_{1}-\delta, \bar{p}_{1}+\delta\right)$ for some small $\delta$. Since $u_{2}^{*}$ is monotone increasing in $p_{2}$, we have $u_{2}^{*}\left(p_{1}, p_{2}\right) \geq C-1$ in $\left(\bar{p}_{1}-\delta, \bar{p}_{1}+\delta\right) \times(1-\varepsilon, 1)$. This shows that

$$
\lim _{\left(p_{1}, p_{2}\right) \rightarrow\left(\bar{p}_{1}, 1\right)} u_{2}^{*}\left(p_{1}, p_{2}\right)=+\infty
$$

for any $\bar{p}_{1} \in \mathbb{R}$, and in particular, $u_{2}^{*}$ is positive near the point $(2,1)$. Without loss of generality, we may assume that $u_{2}^{*}$ is positive in $[1,3] \times[0,1)$. For any $C>0$ large, we let

$$
v\left(p_{1}, p_{2}\right):=u_{2}^{*}\left(p_{1}, p_{2}\right)-C p_{2}\left(p_{1}-1\right)\left(3-p_{1}\right)-\frac{C}{3} p_{2}^{3}+\frac{C}{3} .
$$


Since $\Delta u_{2}^{*}=0$, it follows that $\Delta v=0$. By the maximum principle, $v \geq 0$ in $[1,3] \times[0,1)$. In particular, $v\left(2, \bar{p}_{2}\right) \geq 0$ where $\bar{p}_{2} \in(0,1)$ is chosen such that

$$
\bar{p}_{2}+\bar{p}_{2}^{3} / 3-1 / 3=1 / 2 .
$$

Hence, $u_{2}^{*}\left(2, \bar{p}_{2}\right) \geq C / 2$ for all $C>0$, which is a contradiction.

\section{Homogenous Grushin type equations}

Let $\Omega$ be a bounded domain in $\mathbb{R}^{n}$ with $C^{2}$ boundary $\partial \Omega$ such that $\Omega \cap\left\{x \mid x_{1}=0\right\} \neq \emptyset$. Consider

$$
L u:=u_{x_{1} x_{1}}+\left|x_{1}\right|^{\alpha} u_{x_{2} x_{2}}=0 \text { in } \Omega,
$$

where $\alpha>-1$. We will see later that the partial Legendre transform of solutions of (2) satisfies (5). Also, (5) appears in [7] in extension formulations for fractional Laplacian operators.

Definition 3.1. We say a function $u$ is a strong solution of (5) if $u \in C^{1}(\Omega) \cap C^{2}\left(\Omega \backslash\left\{x_{1}=0\right\}\right)$ and satisfies

$$
L u=0 \quad \text { in } \Omega \backslash\left\{x_{1}=0\right\} .
$$

In this following, we will see that our definition of strong solution coincides with the classical strong solutions. Indeed, $u \in W_{l o c}^{2, p}$ for any $1 \leq p<-\frac{1}{\alpha}$ if $\alpha \in(-1,0)$, and $u$ is $C^{2, \delta}$ if $\alpha \geq 0$. We have to be careful if we want to study continuous viscosity solutions of (5) which may not have uniqueness property, see Remark 4.3 in [7]. However, $L^{p}$-viscosity solutions of certain elliptic equations with coefficients deteriorating along some lower dimensional manifolds would be such strong solutions, see, e.g., [29]. The following proposition is in the same spirit of Lemma 4.2 in [7]. For regularity properties of solutions of a more general class of quasilinear degenerate elliptic equations we refer to [11].

Proposition 3.2. For any $g \in C(\partial \Omega)$, there exists a unique strong solution $u$ of (5) with $u \in$ $C(\bar{\Omega})$ and $u=g$ on $\partial \Omega$. Furthermore, we have

$$
\max _{\bar{\Omega}} u \leq \max _{\partial \Omega} g, \quad \min _{\bar{\Omega}} u \geq \min _{\partial \Omega} g
$$

and, for any $\Omega^{\prime} \subset \subset \Omega$ and $k \in \mathbb{N}$,

$$
\sum_{l=0}^{k}\left\|\nabla_{x_{2}}^{l} u\right\|_{C^{1}\left(\Omega^{\prime}\right)} \leq C\|g\|_{C^{0}(\partial \Omega)},
$$

where $C>0$ depends only on $n, \alpha, k, \operatorname{dist}\left(\Omega^{\prime}, \partial \Omega\right)$. 
Proof. Uniqueness. Clearly, the uniqueness would follow from (6). The proof of uniqueness in Lemma 4.2 in [7] can be applied to obtain (6) and we include it for completeness. Let $u$ be a strong solution of (5) with $u \in C(\bar{\Omega})$ and $u=g$ on $\partial \Omega$. Let $v=u-\max _{\partial \Omega} g+\varepsilon\left|x_{1}\right|$, where $\varepsilon$ is small. Suppose $v$ has an interior maximum point $\bar{x}$ in $\Omega$. Then $\bar{x}_{1}=0$, since otherwise $v$ satisfies an elliptic equation near $\bar{x}$ which does not allow an interior maximum point. On the other hand, if $\bar{x}_{1}=0$, then $\bar{x}$ can not be a maximum point of $v$ since $\partial_{+} v(\bar{x})>\partial_{-} v(\bar{x})$. Therefore, the maximum of $v$ is achieved on $\partial \Omega$, i.e. $u-\max _{\partial \Omega} g+\varepsilon\left|x_{1}\right| \leq \varepsilon \operatorname{diam}(\Omega)$. Sending $\varepsilon \rightarrow 0$, we obtain $\max _{\bar{\Omega}} u \leq \max _{\partial \Omega} g$. Similarly, we can show that $\min _{\bar{\Omega}} u \geq \min _{\partial \Omega} g$.

Existence. For $\varepsilon>0$ sufficiently small, let $0<\eta_{\varepsilon}\left(x_{1}\right) \in C^{\infty}(-\infty, \infty)$ such that

$$
\eta_{\varepsilon}\left(x_{1}\right)=\left|x_{1}\right|^{\alpha} \quad \text { for } \quad\left|x_{1}\right|>2 \varepsilon ; \quad \eta_{\varepsilon}\left(x_{1}\right)=\varepsilon^{\alpha} \quad \text { for } \quad\left|x_{1}\right| \leq \varepsilon .
$$

By the standard linear elliptic equation theory, there exists a unique solution $u^{\varepsilon} \in C(\bar{\Omega}) \cap$ $C^{\infty}(\Omega)$ of

$$
L_{\varepsilon} u^{\varepsilon}:=u_{x_{1} x_{1}}^{\varepsilon}+\eta_{\varepsilon} u_{x_{2} x_{2}}^{\varepsilon}=0 \text { in } \Omega,
$$

and $u^{\varepsilon}=g$ on $\Omega$. By the maximum principle, we have $\sup _{\Omega}\left|u^{\varepsilon}\right| \leq \sup _{\partial \Omega}|g|$. We will establish proper uniform norms of $u^{\varepsilon}$ and obtain the desired solution by sending $\varepsilon \rightarrow 0$.

Our proof of this part is different from [7] which uses Caffarelli-Gutiérrez's Harnack inequality [5] to obtain uniform interior Hölder norms of those approximating solutions. Instead, we establish an interior bound of $u_{x_{2}}^{\varepsilon}$ first, as in Daskalopoulos-Savin [10]. In view of the standard uniformly elliptic equation theory, we only need to concern about the area near $\left\{x_{1}=0\right\}$. Suppose that $0 \in \Omega$ and $B_{\tau} \subset \Omega$ for some small $\tau>0$. We shall show that $\left\|u_{x_{2}}^{\varepsilon}\right\|_{L^{\infty}\left(B_{\tau / 2}\right)} \leq C$ for some $C$ independent of $\varepsilon$.

We claim that there exists a large universal constant $\beta$ such that

$$
L_{\varepsilon}\left(\beta\left(u^{\varepsilon}\right)^{2}+\left(\varphi u_{x_{2}}^{\varepsilon}\right)^{2}\right) \geq 0 \text { in } \Omega,
$$

where $\varphi$ is some cutoff function in $B_{\tau}$ satisfying $\varphi=1$ in $B_{\tau / 2}, \varphi=0$ in $\Omega \backslash B_{\tau}$, and $\varphi_{x_{1}}=0$ for all $\left|x_{1}\right| \leq \tau / 4$.

Indeed, a simple computation yields

$$
L_{\varepsilon}\left(u^{\varepsilon}\right)^{2}=2\left(\left(u_{x_{1}}^{\varepsilon}\right)^{2}+\eta_{\varepsilon}\left(u_{x_{2}}^{\varepsilon}\right)^{2}\right)
$$

and

$$
\begin{aligned}
L_{\varepsilon}\left(\varphi u_{x_{2}}^{\varepsilon}\right)^{2}= & L_{\varepsilon} \varphi^{2}\left(u_{x_{2}}^{\varepsilon}\right)^{2}+\varphi^{2} L_{\varepsilon}\left(u_{x_{2}}^{\varepsilon}\right)^{2}+2\left(\varphi^{2}\right)_{x_{1}}\left(\left(u_{x_{2}}^{\varepsilon}\right)^{2}\right)_{x_{1}}+2 \eta_{\varepsilon}\left(\varphi^{2}\right)_{x_{2}}\left(\left(u_{x_{2}}^{\varepsilon}\right)^{2}\right)_{x_{2}} \\
= & L_{\varepsilon} \varphi^{2}\left(u_{x_{2}}^{\varepsilon}\right)^{2}+2 \varphi^{2}\left(\left(u_{x_{2} x_{1}}^{\varepsilon}\right)^{2}+\eta_{\varepsilon}\left(u_{x_{2} x_{2}}^{\varepsilon}\right)^{2}\right)+8\left(\varphi_{x_{1}} u_{x_{2}}^{\varepsilon}\right)\left(\varphi u_{x_{2} x_{1}}^{\varepsilon}\right) \\
& +8 \eta_{\varepsilon}\left(\varphi_{x_{2}} u_{x_{2}}^{\varepsilon}\right)\left(\varphi u_{x_{2} x_{2}}^{\varepsilon}\right) .
\end{aligned}
$$

Hence,

$$
\begin{aligned}
L_{\varepsilon}\left(\beta\left(u^{\varepsilon}\right)^{2}+\left(\varphi u_{x_{2}}^{\varepsilon}\right)^{2}\right) \geq & 2 \beta \eta_{\varepsilon}\left(u_{x_{2}}^{\varepsilon}\right)^{2}+2 \varphi^{2}\left(\left(u_{x_{2} x_{1}}^{\varepsilon}\right)^{2}+\eta_{\varepsilon}\left(u_{x_{2} x_{2}}^{\varepsilon}\right)^{2}\right) \\
& +L_{\varepsilon} \varphi^{2}\left(u_{x_{2}}^{\varepsilon}\right)^{2}+8\left(\varphi_{x_{1}} u_{x_{2}}^{\varepsilon}\right)\left(\varphi u_{x_{2} x_{1}}^{\varepsilon}\right)+8 \eta_{\varepsilon}\left(\varphi_{x_{2}} u_{x_{2}}^{\varepsilon}\right)\left(\varphi u_{x_{2} x_{2}}^{\varepsilon}\right) .
\end{aligned}
$$


By the Cauchy inequality and the facts

$$
L_{\varepsilon}\left(\varphi^{2}\right) \geq-C_{1} \eta_{\varepsilon}, \quad\left|\varphi_{x_{1}} u_{x_{2}}^{\varepsilon}\right| \leq C_{1} \eta_{\varepsilon}\left|u_{x_{2}}^{\varepsilon}\right|,
$$

the claim follows for large $\beta$ independent of $\varepsilon$.

By (9) and the maximum principle, we have

$$
\sup _{B_{\tau / 2}}\left|u_{x_{2}}^{\varepsilon}\right| \leq \beta^{1 / 2} \sup _{\Omega}\left|u^{\varepsilon}\right|
$$

Since $L u_{x_{2}}^{\varepsilon}=0$, the same arguments can be applied inductively to show that $\partial^{k} u^{\varepsilon} / \partial x_{2}^{k}$ are bounded in the interior of $\Omega$ for any $k \in \mathbb{Z}^{+}$. Since $\left|u_{x_{2} x_{2}}^{\varepsilon}\right| \leq C$ for some $C$ independent of $\varepsilon$ and $u_{x_{1} x_{1}}^{\varepsilon}+\eta_{\varepsilon} u_{x_{2} x_{2}}^{\varepsilon}=0$, we have

$$
\left|u_{x_{1}}^{\varepsilon}\right| \leq C \int_{-1}^{1} \eta_{\varepsilon}\left(x_{1}\right) \mathrm{d} x_{1}+C
$$

where we used the fact that $u_{x_{1}}^{\varepsilon}$ is bounded uniformly for $B_{3 \tau / 4} \cap\left\{x|| x_{1} \mid \geq \tau / 4\right\}$. Since $\alpha>-1$, the integral $\int_{-1}^{1} \eta_{\varepsilon}\left(x_{1}\right) \mathrm{d} x_{1}$ can be bounded independent of $\varepsilon$. The same arguments would show that $u_{x_{1} x_{2}}^{\varepsilon}$ and $u_{x_{1} x_{2} x_{2}}^{\varepsilon}$ are bounded as well.

For $\alpha \in(-1,0)$ and any point $\bar{x}=\left(\bar{x}_{1}, \bar{x}_{2}\right) \in B_{\tau / 4}$, by the Taylor's formula we have

$$
\begin{aligned}
& u^{\varepsilon}\left(x_{1}, \bar{x}_{2}\right) \\
& =u^{\varepsilon}\left(\bar{x}_{1}, \bar{x}_{2}\right)+u_{x_{1}}^{\varepsilon}\left(\bar{x}_{1}, \bar{x}_{2}\right)\left(x_{1}-\bar{x}_{1}\right)+\left(x_{1}-\bar{x}_{1}\right)^{2} \int_{0}^{1}(1-\lambda) u_{x_{1} x_{1}}^{\varepsilon}\left(\xi_{\lambda}, \bar{x}_{2}\right) \mathrm{d} \lambda \\
& =u^{\varepsilon}\left(\bar{x}_{1}, \bar{x}_{2}\right)+u_{x_{1}}^{\varepsilon}\left(\bar{x}_{1}, \bar{x}_{2}\right)\left(x_{1}-\bar{x}_{1}\right)-\left(x_{1}-\bar{x}_{1}\right)^{2} \int_{0}^{1}(1-\lambda) u_{x_{2} x_{2}}^{\varepsilon}\left(\xi_{\lambda}, \bar{x}_{2}\right) \eta\left(\xi_{\lambda}\right) \mathrm{d} \lambda \\
& =u^{\varepsilon}\left(\bar{x}_{1}, \bar{x}_{2}\right)+u_{x_{1}}^{\varepsilon}\left(\bar{x}_{1}, \bar{x}_{2}\right)\left(x_{1}-\bar{x}_{1}\right)-u_{x_{2} x_{2}}^{\varepsilon}\left(\bar{x}_{1}, \bar{x}_{2}\right)\left(x_{1}-\bar{x}_{1}\right)^{2} \int_{0}^{1}(1-\lambda) \eta\left(\xi_{\lambda}\right) \mathrm{d} \lambda \\
& \quad+O\left(\left|x_{1}-\bar{x}_{1}\right|^{3} \int_{0}^{1} \eta\left(\xi_{\lambda}\right) \mathrm{d} \lambda\right),
\end{aligned}
$$

where $\xi_{\lambda}=\bar{x}_{1}+\lambda\left(x_{1}-\bar{x}_{1}\right)$. One should note that $\int_{0}^{1} \eta\left(\xi_{\lambda}\right) \mathrm{d} \lambda \leq C\left|x_{1}-\bar{x}_{1}\right|^{\alpha}$ for some constant $C>0$ independent of $\varepsilon$. Making use of Taylor's formula again, we have

$$
\begin{aligned}
u^{\varepsilon}\left(x_{1}, x_{2}\right)= & u^{\varepsilon}\left(x_{1}, \bar{x}_{2}\right)+u_{x_{2}}^{\varepsilon}\left(x_{1}, \bar{x}_{2}\right)\left(x_{2}-\bar{x}_{2}\right)+\frac{1}{2} u_{x_{2} x_{2}}^{\varepsilon}\left(\bar{x}_{1}, \bar{x}_{2}\right)\left(x_{2}-\bar{x}_{2}\right)^{2} \\
& +O\left(\left|x_{2}-\bar{x}_{2}\right|^{3}+\left|\left(x_{1}-\bar{x}_{1}\right)\left(x_{2}-\bar{x}_{2}\right)^{2}\right|\right),
\end{aligned}
$$

and

$$
u_{x_{2}}^{\varepsilon}\left(x_{1}, \bar{x}_{2}\right)=u_{x_{2}}^{\varepsilon}\left(\bar{x}_{1}, \bar{x}_{2}\right)+u_{x_{1} x_{2}}^{\varepsilon}\left(\bar{x}_{1}, \bar{x}_{2}\right)\left(x_{1}-\bar{x}_{1}\right)+O\left(\left|x_{1}-\bar{x}_{1}\right|^{2+\alpha}\right) .
$$


Therefore,

$$
\left|u^{\varepsilon}\left(x_{1}, x_{2}\right)-u^{\varepsilon}\left(x_{1}, \bar{x}_{2}\right)-u_{x_{1}}^{\varepsilon}\left(\bar{x}_{1}, \bar{x}_{2}\right)\left(x_{1}-\bar{x}_{1}\right)-u_{x_{2}}^{\varepsilon}\left(\bar{x}_{1}, \bar{x}_{2}\right)\left(x_{2}-\bar{x}_{2}\right)\right| \leq C|x-\bar{x}|^{2+\alpha} .
$$

By the arbitrary choice of $\bar{x}$, we conclude that

$$
\left\|u^{\varepsilon}\right\|_{C^{1,1+\alpha}\left(B_{\tau / 4}\right)} \leq C .
$$

The same argument is also applicable to $\alpha \geq 0$, and one can conclude that

$$
\left\|u^{\varepsilon}\right\|_{C^{2, \delta}\left(B_{\tau / 4}\right)} \leq C
$$

for some $\delta>0$ depending only on $\alpha$.

By passing to a subsequence, we obtain a strong solution $u$ of (5) and $u$ satisfies (7).

Remark 3.3. From the proof of Proposition 3.2 we see that:

- If $\alpha \in(-1,0), u \in C_{l o c}^{1,1+\alpha}(\Omega)$;

- If $\alpha \geq 0, u \in C_{\text {loc }}^{2, \delta}(\Omega)$ for some $\delta>0$ depending only on $\alpha$.

Let

$$
\phi\left(x_{1}, x_{2}\right)=\left|x_{1}\right|^{2+\alpha}+x_{2}^{2} \quad \text { in } \mathbb{R}^{2} .
$$

Then

$$
\nabla^{2} \phi=\left(\begin{array}{cc}
(2+\alpha)(1+\alpha)\left|x_{1}\right|^{\alpha} & 0 \\
0 & 2
\end{array}\right)
$$

and

$$
\left(\nabla^{2} \phi\right)^{1 / 2}=\left(\begin{array}{cc}
\sqrt{(2+\alpha)(1+\alpha)}\left|x_{1}\right|^{\alpha / 2} & 0 \\
0 & \sqrt{2}
\end{array}\right) .
$$

Hence, $\operatorname{det} \nabla^{2} \phi=c(\alpha)\left|x_{1}\right|^{\alpha}$, where $c(\alpha)=2(\alpha+2)(\alpha+1)>0$. For any $x \in \mathbb{R}^{2}$ and $t>0$, denote

$$
S(x, t)=S_{\phi}(x, t)=\left\{y \in \mathbb{R}^{2} \mid \phi(y)<\ell(y)+t\right\},
$$

where $\ell(y)$ is the support plane of $\phi$ at $(x, \phi(x))$. It is direct to verify

Condition $\mu_{\infty}$ [5]: For any given $\delta_{1} \in(0,1)$, there exists $\delta_{2} \in(0,1)$ such that, for all sections $S$ and all small subsets $E \subset S$,

$$
\frac{|E|}{|S|}<\delta_{2} \quad \text { implies } \quad \frac{\int_{E}\left|x_{1}\right|^{\alpha} \mathrm{d} x}{\int_{S}\left|x_{1}\right|^{\alpha} \mathrm{d} x}<\delta_{1} .
$$


Let

$$
A\left(x_{1}, x_{2}\right)=\left(\begin{array}{cc}
\left|x_{1}\right|^{-\alpha} & 0 \\
0 & 1
\end{array}\right)
$$

Clearly,

$$
B:=\left(\nabla^{2} \phi\right)^{1 / 2} A\left(\nabla^{2} \phi\right)^{1 / 2}=\left(\begin{array}{cc}
(2+\alpha)(1+\alpha) & 0 \\
0 & 2
\end{array}\right),
$$

which is positive definite if $\alpha>-1$. Therefore, we can apply Caffarelli-Gutiérrez's Harnack inequality [5] to obtain the following proposition.

Proposition 3.4. Let $u \geq 0$ be a strong solution of

$$
L u=0 \quad \text { in } S\left(x_{0}, 2\right) \text {, }
$$

where $x_{0}$ is an arbitrary point in $\mathbb{R}^{2}$. Then there exists a positive constant $\beta$ depending only on $\alpha$ such that

$$
\sup _{S\left(x_{0}, 1\right)} u \leq \beta \inf _{S\left(x_{0}, 1\right)} u \text {. }
$$

Corollary 3.5. Let $u$ be a strong solution of

$$
L u=0 \quad \text { in } S(0,2) .
$$

Then there exist constants $C>0$ and $\gamma \in(0,1)$ depending only on $\alpha$ such that

$$
\|u\|_{C^{\gamma}(S(0,1))} \leq C\|u\|_{L^{\infty}(S(0,2))} .
$$

Theorem 3.6. Let $u$ be a nonnegative strong solution of

$$
L u=0 \quad \text { in } \mathbb{R}^{2} .
$$

Then $u$ is a constant in $\mathbb{R}^{2}$.

Proof. Consider the scaling $u_{r}=\frac{1}{r} u\left(r^{1 /(2+\alpha)} x_{1}, r^{1 / 2} x_{2}\right)$ for $r>0$. Then $u_{r}$ also satisfies (14). By Proposition 3.4, we have

$$
\sup _{S(0,2)} u_{r} \leq \beta u_{r}(0)
$$

It follows from Corollary 3.5 that

$$
\left[u_{r}\right]_{C^{\gamma}(S(0,1))} \leq C \beta u_{r}(0) .
$$

For any two distinct points $x, y$ in $\mathbb{R}^{2}$, we have, for sufficiently large $r$,

$$
\begin{aligned}
|u(x)-u(y)| & =r\left|u_{r}\left(r^{-1 /(2+\alpha)} x_{1}, r^{-1 / 2} x_{2}\right)-u_{r}\left(r^{-1 /(2+\alpha)} y_{1}, r^{-1 / 2} y_{2}\right)\right| \\
& \leq r\left[u_{r}\right]_{C^{\gamma}(S(0,1))}\left|r^{-2 /(2+\alpha)}\left(x_{1}-y_{1}\right)^{2}+r^{-1}\left(x_{2}-y_{2}\right)^{2}\right|^{\gamma / 2} \\
& \leq C \beta u(0)\left|r^{-2 /(2+\alpha)}\left(x_{1}-y_{1}\right)^{2}+r^{-1}\left(x_{2}-y_{2}\right)^{2}\right|^{\gamma / 2} .
\end{aligned}
$$

Sending $r \rightarrow \infty$, we obtain $u(x)=u(y)$. The proof is completed. 


\section{Regularity for solutions of degenerate Monge-Ampère equations}

Define the measure $\mu_{\alpha}$ in $\mathbb{R}^{2}$ as $\mathrm{d} \mu_{\alpha}=\left|x_{1}\right|^{\alpha} \mathrm{d} x_{1} \mathrm{~d} x_{2}$ for $\alpha>-1$. For any bounded open convex set $\Omega \subset \mathbb{R}^{2}$, it is clear that the measure $\mu_{\alpha}$ has the doubling property in $\Omega$, i.e., there exists a constant $c_{\alpha}>0$, depending only on $\alpha$ and $\Omega$, such that for any $\left(\bar{x}_{1}, \bar{x}_{2}\right) \in \Omega$ and any ellipsoids $E \subset \mathbb{R}^{2}$ centered at origin with $\left(\bar{x}_{1}, \bar{x}_{2}\right)+E \in \Omega$ there holds

$$
\mu_{\alpha}\left(\left(\bar{x}_{1}, \bar{x}_{2}\right)+E\right) \geq c_{\alpha} \mu_{\alpha}\left(\left(\left(\bar{x}_{1}, \bar{x}_{2}\right)+2 E\right) \cap \Omega\right) .
$$

Consequently, we have the following theorem.

Theorem 4.1. Let $\Omega$ be an open convex set in $\mathbb{R}^{2}$, and $u$ be the generalized solution of

$$
\operatorname{det} \nabla^{2} u(x)=\left|x_{1}\right|^{\alpha} \quad \text { in } \Omega \text {, }
$$

with $u=0$ on $\partial \Omega$. Then $u$ is strictly convex in $\Omega, u \in C_{\text {loc }}^{1, \delta}(\Omega)$ for some $\delta>0$ depending only on $\alpha$. Furthermore, the partial Legendre transform $u^{*}$ of $u$ is a strong solution of

$$
L u^{*}=0 \quad \text { in } T(\Omega),
$$

where the map $T$ is given in (4).

Proof. The strict convexity and the $C^{1, \delta}$ regularity was proved in [2, 3]. Hence, $T$ is continuous and one-to-one, and thus, $T(\Omega)$ is open. Let $u_{k} \in C(\bar{\Omega}) \cap C^{\infty}(\Omega)$ be the solution of

$$
\operatorname{det} \nabla^{2} u_{k}=\eta_{1 / k}\left(x_{1}\right) \text { in } \Omega
$$

with $u_{k}=0$ on $\partial \Omega$, where $\eta_{1 / k}\left(x_{1}\right)$ is the same as the one in the proof of Proposition 3.2 with $\varepsilon=1 / k$. Let

$$
T_{k}: \Omega \rightarrow \mathbb{R}^{2}, \quad\left(x_{1}, x_{2}\right) \mapsto\left(x_{1}, \partial_{2} u_{k}(x)\right),
$$

and $u_{k}^{*}$ be the partial Legendre transform of $u_{k}$. Then $u_{k}^{*}$ satisfies (8). Clearly, up to a subsequence, $u_{k} \rightarrow u$ in $C_{l o c}^{1}(\Omega)$ as $k \rightarrow \infty$. Thus, $\lim _{k \rightarrow \infty} T_{k}(x)=T(x)$ for any $x \in \Omega$, and for any $y \in T(\Omega)$ there exists $\lambda$ sufficiently small such that $B_{\lambda}(y) \subset T(\Omega) \cap T_{k}(\Omega)$ for every large $k$. By the same argument used in proof of Proposition 3.2. we can conclude that $u^{*} \in C^{1}(T(\Omega)) \cap C^{2}\left(T(\Omega) \backslash\left\{x_{1}=0\right\}\right)$ and satisfies $L u^{*}=0$ in $T(\Omega) \backslash\left\{x_{1}=0\right\}$.

Theorem 4.2. Let u be a generalized solution of (2). Then $u$ is strictly convex.

Proof. By the two dimensional Monge-Ampère equation theory, if $u$ is a generalized solution of

$$
\operatorname{det} \nabla^{2} u \geq c_{0}>0 \quad \text { in } \Omega,
$$


where $\Omega$ is an open set in $\mathbb{R}^{2}$, then $u$ is locally strictly convex in $\Omega$. Hence, we only need to consider the situation $\alpha>0$. After subtracting a supporting plane of $u$ at origin, we may assume that

$$
u \geq 0 \quad \text { in } \mathbb{R}^{2} \text { and } u(0)=0 .
$$

Claim: There exists a sufficiently large $R>0$ such that

$$
\min _{\partial B_{R}} u>0 .
$$

Indeed, if not, namely, $\min _{\partial B_{R}} u=0$ for all sufficiently large $R>0$. The strict convexity of $u$ away from $\left\{x_{1}=0\right\}$ implies $u\left(R e_{2}\right)=0$ or $u\left(-R e_{2}\right)=0$, where $e_{2}=(0,1)$. Without loss of generality, we may assume $u\left(R e_{2}\right)=0$. Let

$$
M=\max _{\partial B_{1}} u>0,
$$

and $\Delta$ be the triangle generated by the segment $\left\{\left(x_{1}, 0\right)|| x_{1} \mid \leq 1\right\}$ and the point $R e_{2}$. By the convexity of $u$, we have

$$
M \geq u \quad \text { in } \Delta .
$$

It is clear that the ellipsoid

$$
E=\left\{\left(x_{1}, x_{2}\right): x_{1}^{2}+\frac{1}{R^{2}}\left(x_{2}-R / 4\right)^{2}=\frac{1}{16}\right\}
$$

sits in $\Delta$. Let

$$
u_{R}\left(y_{1}, y_{2}\right)=\frac{1}{R} u\left(y_{1}, R\left(y_{2}+1 / 4\right)\right)
$$

We have

$$
\operatorname{det} \nabla^{2} u_{R}\left(y_{1}, y_{2}\right)=\left|y_{1}\right|^{\alpha} \quad \text { in } B_{1 / 4},
$$

and $u_{R} \leq \frac{M}{R}$ in $B_{1 / 4}$. Choosing a small constant $\tau>0$, depending only on $\alpha$, such that

$$
S_{\phi}(0, \tau) \subset B_{1 / 4},
$$

where $\phi$ is given in (12). By the comparison principle (see, e.g., [15]),

$$
0 \leq u_{R} \leq \sqrt{c(\alpha)^{-1}}(\phi-\tau)+\max _{\partial S(0, \tau)} u_{R} \text { in } S_{\phi}(0, \tau),
$$

where $c(\alpha)=2(\alpha+2)(\alpha+1)$. In particular,

$$
0 \leq-\sqrt{c(\alpha)^{-1}} \tau+\max _{\partial S(0, \tau)} u_{R} \leq-\sqrt{c(\alpha)^{-1}} \tau+M / R .
$$

That is

$$
R \leq \frac{\sqrt{c(\alpha)} M}{\tau}
$$

which contradicts to the assumption that $R$ can be arbitrarily large.

Thus, (17) holds and we can conclude Theorem 4.2 from Theorem 4.1. 
One might ask if every solution of

$$
\operatorname{det} \nabla^{2} u=\left|x_{1}\right|^{\alpha} \quad \text { in } B_{1} \subset \mathbb{R}^{2}
$$

is strictly convex, where $\alpha>0$. The following example shows that this is not the case.

Example 4.3. It is clear that for every $\alpha>0$ there always exists a positive convex smooth solution $w$ of the $O D E$

$$
\left\{\begin{array}{l}
\frac{\alpha(\alpha+2)}{4} w(t) w(t)^{\prime \prime}-\frac{(\alpha+2)^{2}}{4}\left(w^{\prime}(t)\right)^{2}=1 \\
w(0)=1 \\
w^{\prime}(0)=1
\end{array}\right.
$$

near $t=0$. Then $u=\left|x_{1}\right|^{\frac{\alpha+2}{2}} w\left(x_{2}\right)$ is a generalized solutions of $\operatorname{det} \nabla^{2} u=\left|x_{1}\right|^{\alpha}$ in a small open set in $\mathbb{R}^{2}$. But $u$ is not strictly convex (is smooth for certain $\alpha$, though). By proper scaling and translation we can make the equation holds in $B_{1}$.

Proof of Theorem 1.1] Let $u$ be a generalized solution of (2). It follows from Theorem 4.2 that $u$ is strictly convex, and hence $u$ is smooth away from $\left\{x_{1}=0\right\}$. By Theorem 4.1, we know that $u \in C_{l o c}^{1, \delta}\left(\mathbb{R}^{2}\right)$ and the partial Legendre transform $u^{*}$ of $u$ is a strong solution of

$$
L u^{*}=u_{11}^{*}+\left|p_{1}\right|^{\alpha} u_{22}^{*}=0 \quad \text { in } T\left(\mathbb{R}^{2}\right),
$$

where $u_{i i}^{*}=u_{p_{i} p_{i}}^{*}$ and $T\left(x_{1}, x_{2}\right)=\left(x_{1}, u_{x_{2}}\left(x_{1}, x_{2}\right)\right)=\left(p_{1}, p_{2}\right)$. Moreover, $T$ is continuous and one-to-one.

Given Theorem 3.6 and Proposition 3.4 the rest of the proof is similar to that in Section 2 for $\alpha=0$.

Step 1: Prove the theorem under the assumption: $T\left(\mathbb{R}^{2}\right)=\mathbb{R}^{2}$.

Since $u^{*}$ is convex with respect to $p_{2}$, we have that $u_{22}^{*} \geq 0$. Note that $L u_{22}^{*}=0$ in $\mathbb{R}^{2}$. By Theorem 3.6, $u_{22}^{*} \equiv a$ for some nonnegative constant $a$. By the equation $L u^{*}=0$, we have $u_{11}^{*}=-a\left|p_{1}\right|^{\alpha}$. Hence, $u_{121}^{*} \equiv u_{122}^{*} \equiv 0$ in $\left\{p_{1}>0\right\}$. Consequently, $u_{12}^{*} \equiv b$ in $\left\{p_{1}>0\right\}$ for some constant $b$. It follows from calculus that

$$
u^{*}=-\frac{a}{(\alpha+1)(\alpha+2)}\left|p_{1}\right|^{2+\alpha}+\frac{a}{2} p_{2}^{2}+b p_{1} p_{2}+\ell\left(p_{1}, p_{2}\right)
$$

for some linear function $\ell$ in $\left\{p_{1}>0\right\}$. The same argument applies to $\left\{p_{1}<0\right\}$. Since $u^{*}, u_{2}^{*} \in C^{1}\left(\mathbb{R}^{2}\right)$, (20) holds for all $p \in \mathbb{R}^{2}$. Since $u=\left(u^{*}\right)^{*}, a>0$ and we are done.

Step 2: Prove: $T\left(\mathbb{R}^{2}\right)=\mathbb{R}^{2}$.

We prove it by contradiction. Suppose that there exists $\bar{x}_{1}$ such that $\lim _{x_{2} \rightarrow \infty} u_{2}\left(\bar{x}_{1}, x_{2}\right):=$ $\beta_{2}<\infty$. Then, as in Section 2, $\lim _{x_{2} \rightarrow \infty} u_{2}\left(x_{1}, x_{2}\right)=\beta$ for every $x_{1} \in \mathbb{R}$, and we may assume 
$\beta=1$. Therefore, $T\left(\mathbb{R}^{2}\right)=(-\infty, \infty) \times\left(\beta_{0}, 1\right)$ for some $-\infty \leq \beta_{0}<1$. Since $T$ is one-to-one and $u_{2}^{*}\left(p_{1}, p_{2}\right)=x_{2}$, we have $\lim _{p_{2} \rightarrow 1^{-}} u_{2}^{*}\left(p_{1}, p_{2}\right)=\infty$. The same argument in Section 2 shows that

$$
\lim _{\left(p_{1}, p_{2}\right) \rightarrow\left(\bar{p}_{1}, 1\right)} u_{2}^{*}\left(p_{1}, p_{2}\right)=+\infty
$$

for any $\bar{p}_{1} \in \mathbb{R}$

Case 1: $\alpha \geq 0$.

Without loss of generality, we may assume that $u_{2}^{*}$ is positive in $[1,3] \times[0,1)$. For any $C>0$ large, we let

$$
v\left(p_{1}, p_{2}\right):=u_{2}^{*}\left(p_{1}, p_{2}\right)-C p_{2}\left(p_{1}-1\right)\left(3-p_{1}\right)-\frac{C}{3} p_{2}^{3}+\frac{C}{3} .
$$

It is direct to check that $L v<0$ in $[1,3] \times[0,1)$. By the maximum principle, $v \geq 0$ in $[1,3] \times[0,1)$. In particular, $v\left(2, \bar{p}_{2}\right) \geq 0$ where $\bar{p}_{2} \in(0,1)$ is chosen such that

$$
\bar{p}_{2}+\bar{p}_{2}^{3} / 3-1 / 3=1 / 2 .
$$

Hence, $u_{2}^{*}\left(2, \bar{p}_{2}\right) \geq C / 2$ for all $C>0$, which is a contradiction.

Case 2: $\alpha \in(-1,0)$.

Without loss of generality, we may assume that $u_{2}^{*}$ is positive in $[1 / 2,1] \times[0,1)$. For any $C>0$ large, we let

$$
v\left(p_{1}, p_{2}\right):=u_{2}^{*}\left(p_{1}, p_{2}\right)-C p_{2}\left(p_{1}-1 / 2\right)\left(1-p_{1}\right)-\frac{C}{3} p_{2}^{3}+\frac{C}{3} .
$$

It is direct to check that $L v<0$ in $[1,3] \times[0,1)$. By the maximum principle, $v \geq 0$ in $[1 / 2,1] \times[0,1)$. In particular, $v\left(3 / 4, \bar{p}_{2}\right) \geq 0$ where $\bar{p}_{2} \in(0,1)$ is chosen such that

$$
\bar{p}_{2} / 16+\bar{p}_{2}^{3} / 3-1 / 3=1 / 32 .
$$

Hence, $u_{2}^{*}\left(3 / 4, \bar{p}_{2}\right) \geq C / 32$ for all $C>0$, which is a contradiction.

The proof is completed.

\section{References}

[1] Alexandrov, A.D.: Intrinsic Geometry of Convex Surfaces, OGIZ, Moscow-Leningrad, 1948.

[2] Caffarelli, L.: A localization property of viscosity solutions to the Monge-Ampre equation and their strict convexity, Ann. of Math. (2) 131 (1990), no. 1, 129-134.

[3] Caffarelli, L.: Some regularity properties of solutions of Monge Ampère equation, Comm. Pure Appl. Math. 44 (1991), no. 8-9, 965-969. 
[4] Caffarelli, L.: "Topics in PDEs: The Monge-Ampère equation.” Graduate course. Courant Institute, New York University, 1995.

[5] Caffarelli, L.; Gutiérez, C.: Properties of the solutions of the linearized Monge-Ampère equation, Amer. J. Math. 119 (1997), no. 2, 423-465.

[6] Caffarelli, L.; Li, Y.Y.: An extension to a theorem of Jörgens, Calabi, and Pogorelov, Comm. Pure Appl. Math. 56 (2003), 549-583.

[7] Caffarelli, L.; Silvestre, L.: An extension problem related to the fractional Laplacian, Comm. Partial. Diff. Equ., 32 (2007), 1245-1260.

[8] Calabi, E.: Improper affine hyperspheres of convex type and a generalization of a theorem by $K$. Jörgens, Michigan Math. J. 5 (1958), 105-126.

[9] Cheng, S. Y.; Yau, S. T.: Complete affine hypersurfaces. I. The completeness of affine metrics, Comm. Pure Appl. Math. 39 (1986), no. 6, 839-866.

[10] Daskalopoulos, P.; Savin, O.: On Monge-Ampère equations with homogeneous right-hand sides, Comm. Pure Appl. Math. 62 (2009), no. 5, 639-676.

[11] Guan, P.: Regularity of a class of quasilinear degenerate elliptic equations, Adv. Math. 132 (1997), no. 1, 24-45.

[12] Ferrer, L.; Martínez, A.; Milán, F.: An extension of a theorem by K. Jörgens and a maximum principle at infinity for parabolic affine spheres, Math. Z. 230 (1999), 471-486.

[13] Ferrer, L.; Martínez, A.; Milán, F.: The space of parabolic affine spheres with fixed compact boundary, Monatsh. Math. 130 (2000), no. 1, 19-27.

[14] Guan, P.; Li, Y.Y.: The Weyl problem with nonnegative Gauss curvature, J. Differential Geom. 39 (1994), no. 2, 331-342.

[15] Gutiérrez, C. E.: The Monge-Ampère equation. Progress in Nonlinear Differential Equations and their Applications, 44. Birkhäuser Boston, Inc., Boston, MA, 2001.

[16] Han, Q.; Hong, J.-X.: "Isometric embedding of Riemannian manifolds in Euclidean spaces." American Mathematical Society, Providence, RI, 2006.

[17] Heinz, E.: On Weyl's embedding problem, J. Math. Mech. 11 (1962), 421-454.

[18] Hong, J.; Zuily, C.: Isometric embedding of the 2-sphere with nonnegative curvature in $\mathbb{R}^{3}$, Math. Z. 219 (1995), no. 3, 323-334.

[19] Iaia, J.A.: Isometric embeddings of surfaces with nonnegative curvature in $\mathbb{R}^{3}$, Duke Math. J. 67 (1992), 423-459.

[20] Jörgens, K.: Über die Lösungen der Differentialgleichung $r t-s^{2}=1$, Math. Ann. 127, (1954), $130-134$.

[21] Lewy, H.: On the existence of a closed convex surface realizing a given Riemannian metric, Proc. Nat. Acad. Sci.U.S.A. 24 (1938), 104-106.

[22] Nirenberg, L.: The Weyl and Minkowski problems in differential geometry in the large, Comm. Pure Appl. Math. 6 (1953), 337-394.

[23] Nitsche, J. C. C.: Elementary proof of Bernstein's theorem on minimal surfaces, Ann. of Math. (2) 66 (1957), 543-544.

[24] Pogorelov, A. V. On convex surfaces with a regular metric, Doklady Akad. Nauk SSSR (N.S.) 67 (1949), 791-794.

[25] Pogorelov, A. V. On the regularity of convex surfaces with regular metric, Doklady Akad. Nauk SSSR (N.S.) 66 (1949), 1051-1053.

[26] Pogorelov, A. V.: On the improper convex affine hyperspheres, Geometriae Dedicata 1 (1972), no. $1,33-46$. 
[27] Trudinger, N. S.; Wang, X.-J.: The Bernstein problem for affine maximal hypersurfaces, Invent. Math. 140 (2000), 399-422.

[28] Weyl, H.: ber die Bestimmung einergeschlossen konvexen Flche durch ihr Linienelement, Vierteljahrsschrift Naturforsch. Gesellschaft 61 (1916), 40-72.

[29] Xiong, J.: $C^{1,1}$ estimates for elliptic equations with partial and piecewise continuous coefficients, Methods and Applications of Analysis, 18 (2011), 373-390.

T. Jin

Department of Mathematics, The University of Chicago

5734 S. University Avenue, Chicago, IL 60637, USA

Email: tj@math.uchicago.edu

J. Xiong

Beijing International Center for Mathematical Research, Peking University

Beijing 100871, China

Email: jxiong@math.pku.edu.cn 